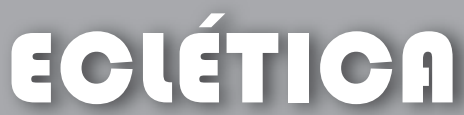 química
}

\section{AVALIAÇÃO DA QUALIDADE DAS ÁGUAS DO RIO SÃO FRANCISCO FALSO, TRIBUTÁRIO DO RESERVATÓRIO DE ITAIPU, PARANÁ.}

\author{
Gilmar. Silvério da Silva, ${ }^{* 1}$ Sandra Miola ${ }^{2}$, Gilberto Silvério da Silva ${ }^{3}$, Eliane Rodrigues de Sousa ${ }^{4}$ \\ ${ }^{1}$ Instituto Federal de Educação, Ciência e Tecnologia do Maranhão, Campus Monte Castelo, 65025-001, São Luís-MA, Brasil. \\ ${ }^{2}$ Universidade Tecnológica Federal do Paraná, Campus Medianeira, 85884-000, Medianeira/PR, Brasil. \\ ${ }^{3}$ Instituto Federal de Educação, Ciência e Tecnologia Goiano, Campus Morrinhos, , BR 153, km 633 - C. Postal 92 - CEP: $75650-00$, \\ Morrinhos/MA. \\ ${ }^{4}$ Instituto Federal de Educação, Ciência e Tecnologia do Maranhão,Campus Maracanã, São Luís-MA, Brasil. \\ *gilmarsilverio@gmail.com
}

\begin{abstract}
Resumo: uma avaliação do impacto das espécies do nitrogênio e do fósforo foi conduzida nas águas do Rio São Francisco Falso, afluente do Reservatório de Itaipu. Os resultados de quatro campanhas revelam que o estado trófico destas águas oscila de oligotróficas a mesotróficos, sendo que o ultimo estado foi observado em campanhas sobre influência de chuvas. Das espécies do nitrogênio, o $\mathrm{NO}_{2}$ apresentou valores acima dos recomendados pela agência ambiental canadense, representando um risco à vida aquática. Em comparação ao Rio Ocoí, outro afluente do Reservatório de Itaipu, as águas do Rio São Francisco Falso apresentam melhor qualidade.
\end{abstract}

Palavra-chave: eutrofização, bacia hidrográfica, índice do Estado Trófico

\section{Introdução}

Fósforo e nitrogênio apresentam-se no meio ambiente segundo várias espécies [1, 2]. O fósforo geralmente é encontrado na coluna d’água na forma de ortofosfato, o qual é prontamente assimilável pelos organismos. O nitrogênio, por sua vez, ocorre como nitrito e nitrato, suas formas oxidadas, e amônia, a forma reduzida. Embora essenciais à produção de biomassa, o excesso destes nutrientes pode levar o corpos aquáticos à eutrofização e suas nefastas conseqüências [3, 4, 5, 6]. Além disso, as espécies do nitrogênio podem ser tóxicas à vida aquática [7]. Com esta preocupação, Silva e Jardim [8] desenvolveram um índice específico para avaliação da toxicidade da amônia para proteção da vida aquática. Silva et. al. [9] chamou atenção para a toxicidade do nitrito em corpos aquáticos.
De um modo geral, os poluentes abordados neste trabalho estão intimamente conectados ao estilo de vida da sociedade [10] e da infra-estrutura de saneamento, tornando-se muitas vezes causa principal de preocupação das comunidades nacional e internacional, como no caso de nova diretiva européia para gestão destes compostos [11]. Nesta direção, o presente trabalho oferece uma avaliação do estado de qualidade das águas destes afluentes do Reservatório de Itaipu, um dos principais ecossistemas nacionais e cujas águas vem apresentando sinais de eutrofização [9]. Especificamente, o trabalho visa avaliar o impacto das espécies do nitrogênio e do fósforo na qualidade das águas do Rio São Francisco Falso, investigando o perfil de fontes poluidoras neste sistema aquático. 
Área de Estudo

O Rio São Francisco Falso pertence à bacia hidrográfica do Paraná III e é um dos afluentes do Reservatório de Itaipu (Figura 1). O presente rio
é formado por dois braços, chamados de Rio São

Figura 1. Reservatório de Itaipu e seus afluentes na bacia do Rio Paraná III. O símbolo · representa os municípios. S.1, S.2, N.1 e N.2 correspondem aos pontos de coleta do braço sul e norte do Rio São Francisco Falso, respectivamente.

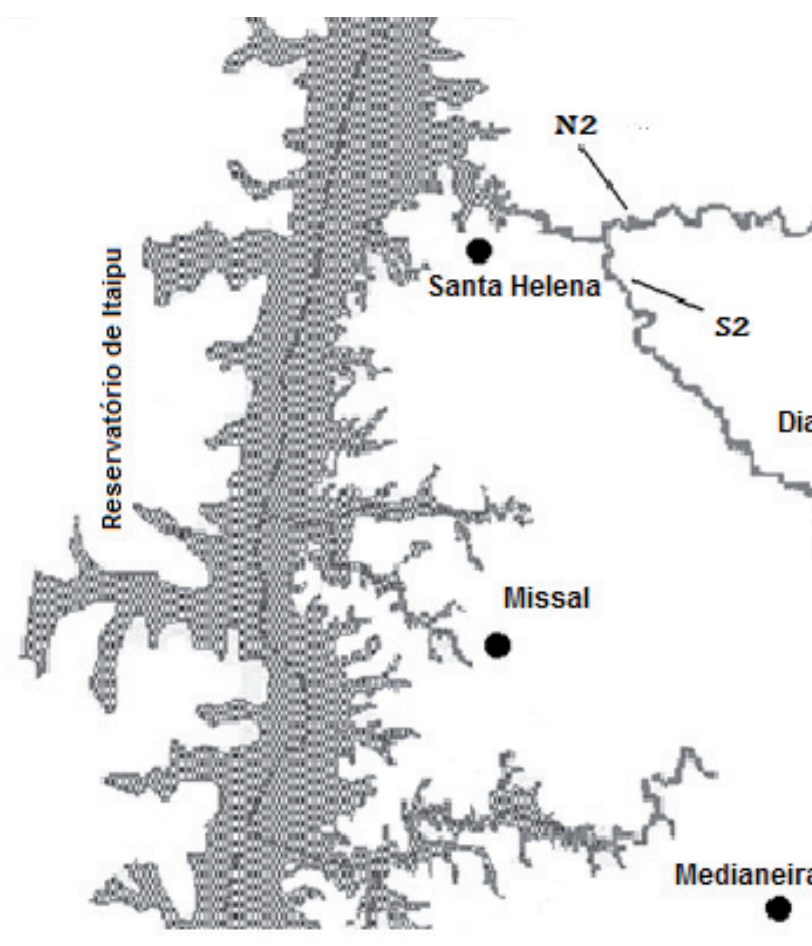

Rio São Francisco Falso Braço Norte

aquático não recebe ( águas, sendo marcado pela presença de atividades de pecuária e agricultura. O presente rio não é manancial para os municípios em seu entorno.

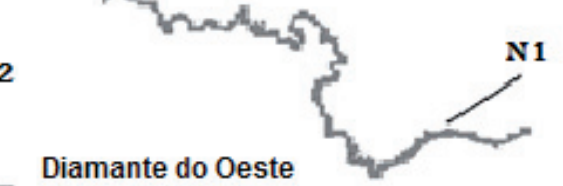

Diamante do Oeste

Rio São Francisco Falso Braço Sul

S1

Vera Cruz do Oeste
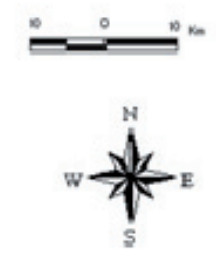

Pontos de coleta e amostragem

Em cada um dos braços do Rio São Francisco Falso foi escolhido dois pontos de amostra, oul tro próximo à foz (Figura 1). No braço norte os pontos foran: N1: S $2459481^{\prime}$ e W 5352 572' (altitude: 447m); N2: S 2452 057' e W 5413 189' (altitude: 225m). No braço sul os pontos fo-
ram: S1: S 2459 569' e W 5407 822' (altitude: 248m); S2: S 2454 184' e W 5412 709' (altitu-

de: $208 \mathrm{~m})$. As coletas foram realizadas durante quatro campanhas durante o ano de 2006: 08/08, $22 / 09,26 / 10$ e $06 / 12$. A segunda campanha foi realizada logo após chuvas, portanto, com grande (2) clacia (campanhas ocorreram na ausência de chuvas, marcado pelo nício de um período de estiagem. Na quarta campanha, o nível d’água estava relativamente alto comparativamente a primeira e terceira campanha devido a chuvas que ocorreram dias antes.

\section{Análises}

As análises realizadas nas amostras de água foram: fósforo total (FT), amônia $\left(\mathrm{NH}_{3}\right)$, nitrito $\left(\mathrm{NO}_{2}\right)$, nitrato $\left(\mathrm{NO}_{3}\right)$, cloreto $(\mathrm{Cl})$, turbidez, $\mathrm{pH}$, condutividade (cond.). As concentrações de FT, NO e NO foram expressas como $\mathrm{mg} \mathrm{L}^{-1}$ de F $N \mathrm{~N}_{2}$ e $\mathrm{NO}_{3}$. e $\mathrm{N}$, respectivanente. Os nétodos analíticos utilizados foram estabelecidos pela APHA [12]. A amostras foram coletadas na superfície da colun d'água e acondicionadas seguindo a Norma NBR 9898 ABNT [13], e encaminhadas ao laboratório da UTFPR onde posteriormente foram analisadas.

\section{Índice do Estado Trófico (IET)}

Para avaliar o estado trófico das águas do Rio São Francisco Falso foi utilizado o índice desenvolvido por Carlson $[14,15]$ e recentemente adaptado para águas dos rios do Estado de São Paulo por Lamparelli [16]. Este índice pode ser aplicado usando a concentração de FT e de clorofila $a$; este trabalho aplicou somente o primeiro parâmetro e, neste caso, o índice é chamado de índice do estado trófico baseado no FT (IETp) [17] segundo a equação 1 . IETp indica a capacidade potencial do corpo aquático em produzir biomassa em excesso, enquanto o índice composto pela conex exa de clor flla a mede a produça efetiva de bişassa produch [16]. Detahes sobre de biomassa produzida [16]. Detathes sobre IETp e grau de trofia é discutido em detalhe na literatura $[16,17]$.

$\operatorname{IETp}=10\left(6-\left(1,77-0,42 \frac{\ln F \mathrm{~T}}{\ln 2}\right)\right)$ guas do Rio São Frani 1. Deve-se destacar que gunda campanha foi marcada pela presença de chuvas e o nivel das águas estava muito elevado. Nesta campanha, os valores de alguns parâmetros mostraram-se expressivamente maiores. Po exemplo, o $\mathrm{Cl}$ passou de 6,4 $\mathrm{mg} \mathrm{L}^{-1}$ na primeira campanha para $19,2 \mathrm{mg} \mathrm{L}^{-1}$ no ponto $\mathrm{S} 1$ na segunda campanha. Em geral, os valores dos parâmetros mantiveram-se dentro do limite estabelecido pela Portaria 357/05 para corpos aquáticos

No caso das espécies do nitrogênio, $\mathrm{NO}_{2}$ $\mathrm{NO}_{3}$ e $\mathrm{NH}_{3}$, na segunda campanha foram observadas uma elevação das suas concentrações, o que se explica pela contribuição da bacia de drenagem, cujo escoamento superficial contribui para o transporte destas espécies das áreas dedicadas a agricultura e pecuária, em particular de granjas de suínas, até os corpos aquáticos. Durante os períodos chuvosos, os escoamentos das águas pluviais transportam inímeras espécies qúmicas, entre as quais $\mathrm{NO}_{2}$ e $\mathrm{NO}_{3}$, as quais são muito solúveis em água [19]. No caso da $\mathrm{NH}_{3}$, a sua concentração na segunda campanha atingiu $0,44 \mathrm{mg} \mathrm{L}^{-1}$ no ponto N1. Considerando que o $\mathrm{pH}$ destas águas encontra-se em 7, segundo prescreve a Diretiva Conama 357/05, o limite para esta espécie é de $3.7 \mathrm{mg} \mathrm{L}^{-1} \mathrm{NH}_{3}-\mathrm{N}$ para $\mathrm{pH} £ 7.5$ [18]. Portanto, devido ao baixo valor de $\mathrm{NH}_{3}$ encontrado nestas amostras, pode-se concluir que tanto a presença de fontes pontuais quanto difusas parecem não ser tão expressivas, sendo similar ao máximo valor de $\mathrm{NH}_{3}$ observado para as águas do Rio Ocoí, 0,56 $\mathrm{NH}_{3}-\mathrm{N}[9]$.

Além da $\mathrm{NH}_{3}$, outra espécie do nitrogênio importante para proteção da vida aquática é o $\mathrm{NO}_{2}$. De acordo com Canadian Water Quality Guidelines (CCME, 2001) [20], o valor de $\mathrm{NO}_{2}$ estabelecido para proteção da vida aquática é de $0,06 \mathrm{mg} \mathrm{L}^{-1} \mathrm{NO}_{2}-\mathrm{N}$. No mesmo documento é estaque está um limite de $13 \mathrm{mg} \mathrm{L}^{-1} \mathrm{~N}^{-}-\mathrm{N}$, $\mathrm{NO}$. que está espécie pode ser convertido mais altos valores de $\mathrm{NO}_{2} \mathrm{~N}_{3}$ $0,32 \mathrm{mg} \mathrm{L}^{-1}$, respectivamente. Os valores para o $\mathrm{NO}_{2}$ observados em todos os pontos e campanhas superam o valor-guia do CCME e representa um risco para a vida aquática. Neste cenário, atenção especial deve ser dedicada à monitoração destas águas diante uso potencial de suas águas para aqüicultura. 
Tabela 1. Parâmetros ambientais das águas do Rio São Francisco Falso. As quatro campanhas foram realizadas no segundo semestre de 2006.

\begin{tabular}{|c|c|c|c|c|}
\hline \multirow{2}{*}{ Parâmetros } & \multicolumn{4}{|c|}{ Campanhas } \\
\hline & $1^{\mathrm{a}}$ & $2^{\mathrm{a}}$ & $3^{\text {a }}$ & $4^{\mathrm{a}}$ \\
\hline \multicolumn{5}{|l|}{ *S.1 Point } \\
\hline $\mathrm{Cl}$ & 6,4 & 19,2 & 17,04 & 15,4 \\
\hline Condutividade & 61,7 & 98 & 66,3 & 83 \\
\hline $\mathrm{pH}$ & 7,1 & 7,0 & 6,9 & 7,2 \\
\hline Turbidez & 2,67 & 71 & 6,0 & 80 \\
\hline $\mathrm{NO}_{2}$ & 0,10 & 0,21 & 0,08 & 0,13 \\
\hline $\mathrm{NO}_{3}$ & 0,22 & 0,32 & 0,12 & 0,22 \\
\hline $\mathrm{NH}_{3}$ & 0,08 & 0,22 & - & - \\
\hline \multicolumn{5}{|l|}{ *S.2 Point } \\
\hline $\mathrm{Cl}$ & 5,30 & 17,8 & 16,33 & 46,5 \\
\hline Condutividade & 72,4 & 102 & 73,6 & 78 \\
\hline $\mathrm{pH}$ & 7,2 & 6,9 & 6,9 & 7,3 \\
\hline Turbidez & 4,17 & 69,2 & 5,74 & 17,6 \\
\hline $\mathrm{NO}_{2}$ & 0,13 & 0,20 & 0,12 & 0,15 \\
\hline $\mathrm{NO}_{3}$ & 0,19 & 0,29 & 0,17 & 0,25 \\
\hline $\mathrm{NH}_{3}$ & 0,09 & 0,23 & - & - \\
\hline \multicolumn{5}{|l|}{ *N.1 Point } \\
\hline $\mathrm{Cl}$ & 4,9 & 16,3 & 17,75 & 16,3 \\
\hline Condutividade & 52,8 & 105 & 44,4 & 65 \\
\hline $\mathrm{pH}$ & 7,4 & 7,0 & 7,0 & 6,9 \\
\hline Turbidez & 6,13- & 50,3 & 8 & 30,2 \\
\hline $\mathrm{NO}_{2}$ & 0,15 & 0,19 & 0,10 & 0,16 \\
\hline $\mathrm{NO}_{3}$ & 0,25 & 0,30 & 0,15 & 0,27 \\
\hline $\mathrm{NH}_{3}$ & 0,09 & 0,43 & - & - \\
\hline \multicolumn{5}{|l|}{ *N.2 Point } \\
\hline $\mathrm{Cl}^{-}$ & 6,4 & 13,5 & 17,75 & 17,8 \\
\hline Condutividade & 54,3 & 85 & 57,4 & 60 \\
\hline $\mathrm{pH}$ & 7,4 & 6,9 & 7,1 & 7,1 \\
\hline Turbidez & 5,20 & 9,35 & 9,35 & 27,2 \\
\hline $\mathrm{NO}_{2}$ & 0,19 & 0,18 & 0,11 & 0,14 \\
\hline $\mathrm{NO}_{3}$ & 0,24 & 0,27 & 0,19 & 0,24 \\
\hline $\mathrm{NH}_{3}$ & 0,17 & 0,11 & - & - \\
\hline
\end{tabular}

Falso, classe 2, sistema aquático lótico que deságua diretamente em ambiente lêntico, o reservatório de Itaipu, é de $0,05 \mathrm{mg} \mathrm{L}^{-1} \mathrm{P}$ [18]. Logo, as águas deste sistema aquático estão abaixo deste águas deste sistema aquátí estão abaixo deste para fico (IET) , as anostras se enquadram em sua maioria como oligoto risco de eutrofização (Tabela 2). Os pontos que foram enquadrados como mesotróficos correspondem à segunda campanha, devido à presença de chuvas, e a quarta campanha, cujo nível d’água estava relativamente alto comparativamente a primeira e terceira campanha. Esta piora da qualidade da água com a presença de chuvas reforça o fato de que a principal causa de poluição destas lato dire a principal casua de poluição destas

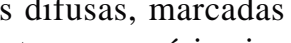
pelo uso intensivo de fertilizantes e pecu
tensivo, especialmente aves e suínos. giu $0,1 \mathrm{mg}$ $357 / 05$, os valores de FT para o Rio Sã

Figura 2. Perfil espacial-temporal da concentração de fósforo total (FT) nas águas do Rio São Francisco Falso, braços norte e sul, Tributário do Reservatório de Itaipu, Paraná.

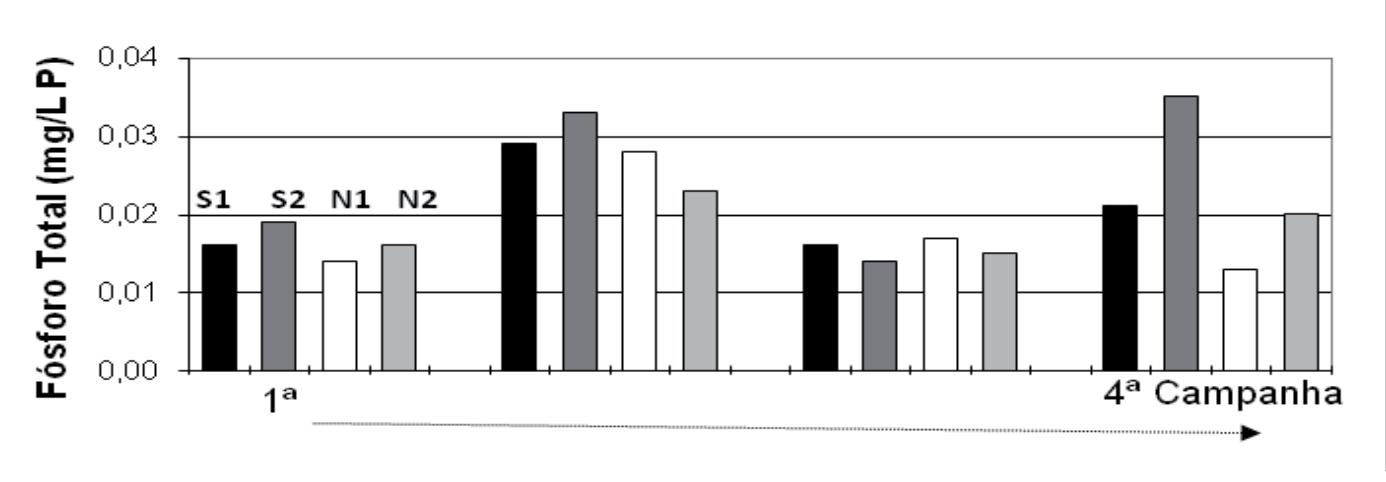

Tabela 2. Índice do Estado Trófico baseado no fósforo (IETp) para as amostras de águas do Rio São Francisco Falso, Tributário do Reservatório de Itaipu, Paraná.

\begin{tabular}{ccccccccc}
\hline \multirow{2}{*}{$\begin{array}{c}\text { Ponto } \\
\text { coleta }\end{array}$} & \multicolumn{2}{c}{$1^{\mathrm{a}}$ Campanha } & \multicolumn{2}{c}{$2^{\mathrm{a}}$ Campanha } & \multicolumn{2}{c}{$3^{\mathrm{a}}$ Campanha } & \multicolumn{2}{c}{$4^{\mathrm{a}}$ Campanha } \\
\cline { 2 - 8 } & IETp & Classe & IETp & Classe & IETp & Classe & IETp & Classe \\
\hline S1 & 48,3 & Oligo $^{\mathrm{a}}$ & 51,4 & Oligo & 48,3 & Oligo & 49,8 & Oligo \\
S2 & 49,2 & Oligo & 52,1 & Meso $^{\mathrm{b}}$ & 47,7 & Oligo & 52,4 & Meso \\
N1 & 47,7 & Oligo & 51,3 & Oligo $^{2}$ & 48,7 & Oligo & 47,3 & Oligo \\
N2 & 48,3 & Oligo & 50,2 & Oligo & 48,1 & Oligo & 49,5 & Oligo \\
\hline
\end{tabular}

oligotrofico, ${ }^{\mathrm{b}}$ mesotrofico 


\section{Conclusão}

As águas do Rio São Francisco Falso não apresentaram condições propícias à produção de biomassa, uma vez que prevaleceu em suas águas o estado oligotrófico. As espécies de nitrogênio e o fósforo total tiveram suas concentrações elevadas durante campanhas marcadas pela influência de chuvas, indicando presença de fontes difusas. $\mathrm{O} \mathrm{NO}_{2}$ em todas as amostras apresentou valores de concentração acima do recomendado pela CCME para proteção da via aquática. Por sua vez, a $\mathrm{NH}_{3}$ não representa um risco potencial à vida aquática em função das baixas concentrações. Em comparação com o Rio Ocoí, o Rio São Francisco Falso demonstra estar em melhor condição de preservação, o que pode ser em parte explicado pela ausência de atividades urbano-industriais.

\section{Agradecimentos}

Os autores agradecem a Fundação Parque Tecnológico de Itaipu (FPTI) e UTFPR/Campus Medianeira pelo apoio financeiro.

Abstract: an environmental assessment about phosphorus and nitrogen species was carried out in waters of São Francisco Falso River, tributary of Itaipu Reservoir. Results from four field campaigns showed that trophic state vary of oligotrophic to mesotrophic, being the latter observed under rain influence. Among nitrogen species, $\mathrm{NO}_{2}$ presented concentration above guide-value recommended by Canadian environmental agency, what means a risk for aquatic life. In comparison to Ocoí River, another tributary of Itaipu Reservoir, São Francisco’s waters showed better quality.

Keyword: eutrophication, hydrographic basin, trophic state index.

\section{Referências}

[1] Koo, B. K., 0’Connell, P. E., Sci. Total Environ. 359 (2006) 1.

[2] Spivakov, B. YA., Maryutina T. A., Muntau, H., Puré AppI. Chem. 71 (1999) 2161.

[3] Kyllmar, K., Carisson, C., Gustafson, A., Ulén, B., Johnsson, H., Agr Ecosyst Environ., 115 (2006) 15.

[4] United States Environmental Protection Agency, USEPA; Nutrient Criteria-Technical Guidance Manual- Rivers and Streams, Washington, D.C, 2000, http://www.epa.gov/ waterscience/criteria/nutrient/guidance/rivers/index.html, acessado em Junho 2009.

[5] United States Environmental Protection Agency (USEPA); Protocol for Developing Nutrient TMDLs -EPA 841-B-99007, Washington D.C., 1999. http://www.epa.gov/owow/ tmdl/nutrient/pdf/nutrient.pdf, acessado em Julho 2008.

[6] Falconeri, I. R., Humpage, A . R., Int. J. Environ. Res. Public Health 2 (2005) 43.

[7] Hooda, P. S., Moynagh, M., Svoboda, L. F., Thurlow, M., Stewart, M., Thomson, M., Anderson, H.A., Sci. Total Environ. 201 (1997) 63.

[8] Silva, G. S., Jardim, W. F., Quím. Nova 29 (2006) 689.

[9] Silva, G. S., Silva, G. S., Sousa, E. R., Konrad, Cristiane., Bem, C. C., Pauli, J., Pereira, A, J. Braz. Chem. Soc., 20: 9 (2009) 1580.

[10] Chapman, A S., Foster, l. D., Lees, J. A., Hodgkinson, R. A., Jackson, R. H., Sci. Total Environ. 66 (2001) 95.
[11] E.E.C. (European Economic Community); Directive 91/271/EEC, on Urban waste water treatment, 1991. http:// eur-lex.europa.eu/pt/index.htm, acessado em Junho 2009.

[12] American Public Health Association, APHA; Standard Methods for the Examination of Water and Wastewater. 16. ed., American Water Works Association and Water Pollution Control Federation: Washington, DC, 1985.

[13] Associação Brasileira de Normas Técnicas, ABNT; NBR 9898: Preservação e técnicas de amostragem de efluentes líquidos e corpos receptores, Rio de Janeiro, 1987.

[14] Carlson, R. E., Limnol. Oceanogr. 22 (1997) 361.

[15] Toledo Jr., A. P., Agudo, E. G., Talarico, M., Chinez, S. J. XIX Congresso Interamericano de Engenharia Sanitária Ambiental- AIDIS, Santiago do Chile, Chile, 1984.

[16] Lamparelli, M. C. 2004. Grau de trofia em corpos d'água do estado de São Paulo: avaliação dos métodos de monitoramento - São Paulo, p. 238. Tese (Doutorado) Instituto de Biociências da Universidade de São Paulo, Brasil, 2004.

[17] Companhia de Tecnologia de Saneamento Ambiental, CETESB; Relatório de Qualidade das Águas Interiores do Estado de São Paulo, São Paulo, 2004.

[18] Portaria 365/05, http://www.mma.gov.br/port/conama, accessed in February de 2009.

[19] Jarvie, H. P., Whitton, B. A., Neal. C., Sci. Total Environ. 210/211 (1998) 79.

[20] http://www.ccme.ca/ acessado em Fevereiro 2009. 